PROCEEDINGS OF THE

AMERICAN MATHEMATICAL SOCIETY

Volume 130, Number 12, Pages 3719-3724

S 0002-9939(02)06517-6

Article electronically published on May 15, 2002

\title{
WHITTLE TYPE INEQUALITY FOR DEMISUBMARTINGALES
}

\author{
B. L. S. PRAKASA RAO
}

(Communicated by Claudia M. Neuhauser)

\begin{abstract}
A Whittle type inequality for demisubmartingales is derived and a strong law of large numbers for functions of a demisubmartingale is obtained.
\end{abstract}

\section{INTRODUCTION}

Whittle ([12]) proved an inequality for real valued random variables generalizing the Kolmogorov inequality, the inequality of Hajek-Renyi ([3]) and the inequality of Dufresnoy ([2]). An application of this result for Hilbert space valued random elements $\left\{Z_{k}, k \geq 1\right\}$ such that the family $\left\{\phi_{k}\left(Z_{k}\right), k \geq 1\right\}$ is a real valued submartingale is given in Rao $([\underline{8})$. An application of this result to obtain a lower bound for the probability of a simultaneous confidence region in multivariate analysis is given in Rao (8]) sharpening the bound given in Sen ([10]). Recently Shixin (11]) proved a Hajek-Renyi type inequality for Banach space valued martingales. A Whittle type inequality for Banach space valued martingales was given in Prakasa Rao ([6]) from which the results in Shixin ([11) follow as special cases.

We now derive a Whittle type inequality for demisubmartingales. This result generalises the recent results on Hajek-Renyi type inequality for demimartingales proved by Christofides ([1]) and the Hajek-Renyi type inequality for associated sequences proved by Prakasa Rao ([5]).

\section{Preliminaries}

Let $S_{i}, i \geq 1$, be a sequence of integrable random variables such that

$$
E\left\{\left(S_{j+1}-S_{j}\right) f\left(S_{1}, \ldots, S_{j}\right)\right\} \geq 0, j \geq 1,
$$

for every componentwise nondecreasing function $f$ such that the expectation is defined. Then the sequence $\left\{S_{j}, j \geq 1\right\}$ is called a demimartingale (cf. Newman and Wright (4 $)$ ). If condition (2.1) holds for every componentwise nonnegative nondecreasing function $f$ such that the expectation is defined, then the sequence $\left\{S_{j}, j \geq 1\right\}$ is called a demisubmartingale.

A collection of random variables $X_{i}, 1 \leq i \leq n$, is said to be associated if

$$
\operatorname{Cov}\left(f\left(X_{1}, \ldots, X_{n}\right), g\left(X_{1}, \ldots, X_{n}\right)\right) \geq 0
$$

Received by the editors June 15, 2001 and, in revised form, August 3, 2001.

2000 Mathematics Subject Classification. Primary 60E15.

Key words and phrases. Whittle's inequality, Kolmogorov's inequality, maximal inequality, Hajek-Renyi inequality, Dufresnoy's inequality, demisubmartingale, demimartingale, associated sequences, strong law of large numbers. 
for any two componentwise nondecreasing functions $f$ and $g$ such that the covariance exists. An infinite sequence of random variables $\left\{X_{n}, n \geq 1\right\}$ is said to be associated if every finite subset of $\left\{X_{n}, n \geq 1\right\}$ is associated.

If $X_{i}, 1 \leq i \leq n$, is an associated sequence of random variables with $E\left(X_{i}\right)=$ $0,1 \leq i \leq n$, then the sequence of partial sums $S_{i}=X_{1}+\cdots+X_{i}, 1 \leq i \leq n$, forms a demimartingale (cf. Newman and Wright [4).

For an extensive review of the probabilistic properties of associated sequences of random variables and related statistical inference problems, see Prakasa Rao and Dewan ([7) and Roussas ([9]).

\section{WhitTle tyPe INEQUALity}

Let $S_{n}, n \geq 1$, be a demisubmartingale and $\phi($.$) be a nondecreasing convex$ function. Then the sequence $\phi\left(S_{n}\right), n \geq 1$, is a demisubmartingale by Lemma 2.1 of Christofides ([1]).

We now state our main theorem.

Theorem 3.1. Let the sequence of random variables $\left\{S_{n}, n \geq 1\right\}$ be a demisubmartingale and $\phi($.$) be a nonnegative nondecreasing convex function such that \phi\left(S_{0}\right)$ $=0$. Let $\psi(u)$ be a positive nondecreasing function for $u>0$. Let $A_{n}$ be the event that $\phi\left(S_{k}\right) \leq \psi\left(u_{k}\right), 1 \leq k \leq n$, where $0=u_{0}<u_{1} \leq \cdots \leq u_{n}$. Then

$$
P\left(A_{n}\right) \geq 1-\sum_{k=1}^{n} \frac{E\left[\phi\left(S_{k}\right)\right]-E\left[\phi\left(S_{k-1}\right)\right]}{\psi\left(u_{k}\right)} .
$$

If, in addition, there exist nonnegative real numbers $\Delta_{k}, 1 \leq k \leq n$, such that

$$
\begin{aligned}
0 & \leq E\left[\left(\phi\left(S_{k}\right)-\phi\left(S_{k-1}\right)\right) f\left(\phi\left(S_{1}\right), \ldots, \phi\left(S_{k-1}\right)\right)\right] \\
& \leq \Delta_{k} E\left[f\left(\phi\left(S_{1}\right), \ldots, \phi\left(S_{k-1}\right)\right)\right], 1 \leq k \leq n,
\end{aligned}
$$

for all componentwise nonnegative nondecreasing functions $f$ such that the expectation is defined and

$$
\psi\left(u_{k}\right) \geq \psi\left(u_{k-1}\right)+\Delta_{k}, 1 \leq k \leq n,
$$

then

$$
P\left(A_{n}\right) \geq \prod_{k=1}^{n}\left(1-\frac{\Delta_{k}}{\psi\left(u_{k}\right)}\right) .
$$

Remarks. The above result is an analogue of the inequality in Whittle ([12]) for real valued random variables. A version of Theorem 3.1 for a sequence of Hilbert space valued random elements was proved in Rao ([8]) and an application to Banach space valued martingales is given in Prakasa Rao ([6]).

Proof. Since the sequence $\left\{S_{n}, n \geq 1\right\}$ is a demisubmartingale by hypothesis and the function $\phi($.$) is a nondecreasing convex function, it follows that the sequence$ $\left\{\phi\left(S_{n}\right), n \geq 1\right\}$ forms a demisubmartingale by Lemma 2.1 of Christofides ([1). Hence

$$
E\left\{\left(\phi\left(S_{n+1}\right)-\phi\left(S_{n}\right)\right) f\left(\phi\left(S_{1}\right), \ldots, \phi\left(S_{n}\right)\right)\right\} \geq 0, n \geq 1,
$$

for every nonnegative componentwise nondecreasing function $f$ such that the expectation is defined.

Let $\chi_{j}$ be the indicator function of the event $\left[\phi\left(S_{j}\right) \leq \psi\left(u_{j}\right)\right]$ for $1 \leq j \leq n$. 
Note that

$$
\chi_{n} \geq\left(1-\frac{\phi\left(S_{n}\right)}{\psi\left(u_{n}\right)}\right)
$$

and hence

$$
\begin{aligned}
P\left(A_{n}\right) & =E\left(\prod_{i=1}^{n} \chi_{i}\right)=E\left(\left\{\prod_{i=1}^{n-1} \chi_{i}\right\} \chi_{n}\right) \\
& \geq E\left(\left\{\prod_{i=1}^{n-1} \chi_{i}\right\}\left(1-\frac{\phi\left(S_{n}\right)}{\psi\left(u_{n}\right)}\right)\right) .
\end{aligned}
$$

Therefore

$$
\begin{array}{r}
E\left[\left\{\prod_{i=1}^{n-1} \chi_{i}\right\}\left\{\left(1-\frac{\phi\left(S_{n}\right)}{\psi\left(u_{n}\right)}\right)-\left(1-\frac{\phi\left(S_{n-1}\right)}{\psi\left(u_{n}\right)}\right)\right\}+\frac{\phi\left(S_{n}\right)-\phi\left(S_{n-1}\right)}{\psi\left(u_{n}\right)}\right] \\
=E\left[\left(1-\prod_{i=1}^{n-1} \chi_{i}\right)\left(\frac{\phi\left(S_{n}\right)-\phi\left(S_{n-1}\right)}{\psi\left(u_{n}\right)}\right)\right] \geq 0
\end{array}
$$

since the function $1-\prod_{i=1}^{n-1} \chi_{i}$ is a nonnegative componentwise nondecreasing function of $\phi\left(S_{i}\right), 1 \leq i \leq n-1$. Hence

$$
\begin{aligned}
P\left(A_{n}\right) & \geq E\left(\left\{\prod_{i=1}^{n-1} \chi_{i}\right\}\left(1-\frac{\phi\left(S_{n-1}\right)}{\psi\left(u_{n}\right)}\right)\right)-\frac{E\left\{\phi\left(S_{n}\right)\right\}-E\left\{\phi\left(S_{n-1}\right)\right\}}{\psi\left(u_{n}\right)} \\
& \geq E\left(\left\{\prod_{i=1}^{n-2} \chi_{i}\right\}\left(1-\frac{\phi\left(S_{n-1}\right)}{\psi\left(u_{n-1}\right)}\right)\right)-\frac{E\left\{\phi\left(S_{n}\right)\right\}-E\left\{\phi\left(S_{n-1}\right)\right\}}{\psi\left(u_{n}\right)} .
\end{aligned}
$$

The last inequality follows from the observation that the sequence $\psi\left(u_{n}\right), n \geq 1$, is positive and nondecreasing.

Applying this inequality repeatedly, we get that

$$
P\left(A_{n}\right) \geq 1-\sum_{k=1}^{n} \frac{E\left[\phi\left(S_{k}\right)\right]-E\left[\phi\left(S_{k-1}\right)\right]}{\psi\left(u_{k}\right)},
$$

completing the proof of the first part of the theorem.

Note that

$$
\begin{array}{r}
E\left\{\prod_{i=1}^{n-1} \chi_{i}\left(1-\frac{\phi\left(S_{n}\right)}{\psi\left(u_{n}\right)}\right)-\left(1-\frac{\Delta_{n}}{\psi\left(u_{n}\right)}\right)\left(1-\frac{\phi\left(S_{n-1}\right)}{\psi\left(u_{n-1}\right)}\right) \prod_{i=1}^{n-1} \chi_{i}\right\} \\
\geq E\left\{\frac{\phi\left(S_{n-1}\right)}{\psi\left(u_{n}\right) \psi\left(u_{n-1}\right)}\left[\psi\left(u_{n}\right)-\psi\left(u_{n-1}\right)-\Delta_{n}\right] \prod_{i=1}^{n-1} \chi_{i}\right\}
\end{array}
$$

and the last term is nonnegative by hypothesis. Hence

$$
P\left(A_{n}\right) \geq\left(1-\frac{\Delta_{n}}{\psi\left(u_{n}\right)}\right) E\left(\left\{\prod_{i=1}^{n-2} \chi_{i}\right\}\left(1-\frac{\phi\left(S_{n-1}\right)}{\psi\left(u_{n-1}\right)}\right)\right) .
$$

Applying this inequality repeatedly, we obtain that

$$
P\left(A_{n}\right) \geq \prod_{k=1}^{n}\left(1-\frac{\Delta_{k}}{\psi\left(u_{k}\right)}\right)
$$




\section{Applications}

Suppose $\left\{S_{n}, n \geq 1\right\}$ is a demisubmartingale. Then $\left\{\left(S_{n}^{+}\right)^{p}, n \geq 1\right\}$ and $\left\{\left(S_{n}^{-}\right)^{p}\right.$, $n \geq 1\}$ are demisubmartingales by Corollary 2.1 of Christofides (1]). Furthermore $\left|S_{n}\right|^{p}=\left(S_{n}^{+}\right)^{p}+\left(S_{n}^{-}\right)^{p}$ for all $p \geq 1$.

(1) Let $\psi(u)=u^{p}, p \geq 1$. Applying Theorem 3.1, we get that

$$
P\left(S_{j}^{+} \leq u_{j}, 1 \leq j \leq n\right) \geq 1-\sum_{j=1}^{n} \frac{E\left(S_{j}^{+}\right)^{p}-E\left(S_{j-1}^{+}\right)^{p}}{u_{j}^{p}}
$$

and

$$
P\left(S_{j}^{-} \leq u_{j}, 1 \leq j \leq n\right) \geq 1-\sum_{j=1}^{n} \frac{E\left(S_{j}^{-}\right)^{p}-E\left(S_{j-1}^{-}\right)^{p}}{u_{j}^{p}} .
$$

Hence, for every $\varepsilon>0$,

$$
\begin{aligned}
P\left(\sup _{1 \leq j \leq n} \frac{\left|S_{j}\right|}{u_{j}} \geq \varepsilon\right)= & P\left(\sup _{1 \leq j \leq n} \frac{\left|S_{j}\right|^{p}}{u_{j}^{p}} \geq \varepsilon^{p}\right) \\
= & P\left(\sup _{1 \leq j \leq n} \frac{\left(S_{j}^{+}\right)^{p}+\left(S_{j}^{-}\right)^{p}}{u_{j}^{p}} \geq \varepsilon^{p}\right) \\
= & P\left(\sup _{1 \leq j \leq n} \frac{\left(S_{j}^{+}\right)^{p}}{u_{j}^{p}} \geq \frac{1}{2} \varepsilon^{p}\right) \\
& \quad+P\left(\sup _{1 \leq j \leq n} \frac{\left(S_{j}^{-}\right)^{p}}{u_{j}^{p}} \geq \frac{1}{2} \varepsilon^{p}\right) \\
\leq & 2 \varepsilon^{-p} \sum_{j=1}^{n} \frac{E\left(S_{j}^{+}\right)^{p}-E\left(S_{j-1}^{+}\right)^{p}}{u_{j}^{p}} \\
& \quad+2 \varepsilon^{-p} \sum_{j=1}^{n} \frac{E\left(S_{j}^{-}\right)^{p}-E\left(S_{j-1}^{-}\right)^{p}}{u_{j}^{p}} \\
\leq & 2 \varepsilon^{-p} \sum_{j=1}^{n} \frac{E\left|S_{j}\right|^{p}-E\left|S_{j-1}\right|^{p}}{u_{j}^{p}} .
\end{aligned}
$$

In particular for $p=2$, we have

$$
P\left(\sup _{1 \leq j \leq n} \frac{\left|S_{j}\right|}{u_{j}} \geq \varepsilon\right) \leq 2 \varepsilon^{-2} \sum_{j=1}^{n} \frac{E S_{j}^{2}-E S_{j-1}^{2}}{u_{j}^{2}},
$$

which is the Hajek-Renyi type inequality for associated sequences derived in Corollary 2.3 of Christofides ([1]).

Suppose $p=1$. Let $\phi(x)=\max (0, x)$. Then $\phi(x)$ is a nonnegative nondecreasing convex function and it is clear that $S_{n} \leq S_{n}^{+}=\phi\left(S_{n}\right)$ for every $n \geq 1$. Let $\psi(u)=u$. Then

$$
\begin{aligned}
P\left(\sup _{1 \leq j \leq n} \frac{S_{j}}{u_{j}} \geq \varepsilon\right) & \leq P\left(\sup _{1 \leq j \leq n} \frac{S_{j}^{+}}{u_{j}} \geq \varepsilon\right) \\
& \leq \varepsilon^{-1} \sum_{j=1}^{n} \frac{E S_{j}^{+}-E S_{j-1}^{+}}{u_{j}}
\end{aligned}
$$


by Theorem 3.1 which is the Chow type maximal inequality derived in Theorem 2.1 of Christofides ([1]).

(2) Let $p=2$ again in the above discussion. If

$$
E\left(S_{j}^{2}-S_{j-1}^{2}\right) \leq u_{j}^{2}-u_{j-1}^{2}
$$

for $1 \leq j \leq n$, then

$$
P\left(A_{n}\right) \geq \prod_{j=1}^{n}\left(1-\frac{E\left(S_{j}^{2}\right)-E\left(S_{j-1}^{2}\right)}{u_{j}^{2}}\right)
$$

which is an analogue of the Dufresnoy's inequality.

(3) Let $\left\{S_{n}, n \geq 1\right\}$ be a demisubmartingale and $\phi($.$) be a nonnegative nonde-$ creasing convex function such that $\phi\left(S_{0}\right)=0$. Let $\psi(u)$ be a positive nondecreasing function for $u>0$. Then, for any nondecreasing sequence $u_{n}, n \geq 1$ with $u_{0}=0$,

$$
P\left(\sup _{1 \leq j \leq n} \frac{\phi\left(S_{j}\right)}{\psi\left(u_{j}\right)} \geq \varepsilon\right) \leq \varepsilon^{-1} \sum_{k=1}^{n} \frac{E\left[\phi\left(S_{k}\right)\right]-E\left[\phi\left(S_{k-1}\right)\right]}{\psi\left(u_{k}\right)} .
$$

In particular, for any fixed $n \geq 1$,

$$
P\left(\sup _{k \geq n} \frac{\phi\left(S_{k}\right)}{\psi\left(u_{k}\right)} \geq \varepsilon\right) \leq \varepsilon^{-1}\left[E\left(\frac{\phi\left(S_{n}\right)}{\psi\left(u_{n}\right)}\right)+\sum_{k=n+1}^{\infty} \frac{E\left[\phi\left(S_{k}\right)\right]-E\left[\phi\left(S_{k-1}\right)\right]}{\psi\left(u_{k}\right)}\right] .
$$

We now derive a strong law of large numbers for functions of demisubmartingales.

Theorem 4.1. Let $\left\{S_{n}, n \geq 1\right\}$ be a demisubmartingale and $\phi($.$) be a nonnegative$ nondecreasing convex function such that $\phi\left(S_{0}\right)=0$. Let $\psi(u)$ be a positive nondecreasing function for $u>0$ such that $\psi(u) \rightarrow \infty$ as $u \rightarrow \infty$. Further suppose that

$$
\sum_{k=1}^{\infty} \frac{E\left[\phi\left(S_{k}\right)\right]-E\left[\phi\left(S_{k-1}\right)\right]}{\psi\left(u_{k}\right)}<\infty
$$

for a nondecreasing sequence $u_{n} \rightarrow \infty$ as $n \rightarrow \infty$. Then

$$
\frac{\phi\left(S_{n}\right)}{\psi\left(u_{n}\right)} \stackrel{\text { a.s }}{\rightarrow} 0 \text { as } n \rightarrow \infty .
$$

Proof of this result follows by the standard arguments following the inequality (4.5) given above. We omit the details.

\section{REFERENCES}

1. Christofides, T.C.: Maximal inequalities for demimartingales and a strong law of large numbers, Statist. Probab. Lett., 50 (2000), 357-363. MR 2001m:60032

2. Dufresnoy, J.: Autour de l'inegalite de Kolmogorov, C. R. Acad. Sci. Paris, 264A (1967), 603. MR 37:5909

3. Hajek, J. and Renyi, A.: A generalization of an inequality of Kolomogorov, Acta. Math. Acad. Sci. Hung., 6 (1955), 281-284. MR 17:864a

4. Newman, C.M. and Wright, A.L.: Associated random variables and martingale inequalities, Z. Wahrsch. Verw. Geb., 59 (1982), 361-371. MR 85d:60088

5. Prakasa Rao, B.L.S.: Hajek-Renyi type inequality for associated sequences, Statist. Probab. Lett., 2001 (to appear).

6. Prakasa Rao, B.L.S.: Application of Whittle's inequality for Banach space valued martingales, Statist. Probab. Lett., 2001 (to appear).

7. Prakasa Rao, B.L.S. and Dewan, I.: Associated sequences and related inference problems, In Handbook of Statistics: Stochastic Processes: Theory and Methods, (Edited by D.N. Shanbhag and C.R.Rao), North Holland, Amsterdam, 19 (2001), 693-728. CMP 2002:03 
8. Rao, P.: Whittle's inequality in Hilbert space, Theory of Probability and Mathematical Statistics, 16 (1978), 111-116. MR 55:13517]

9. Roussas, G.G.: Positive and negative dependence with some statistical applications, In Asymptotics, Nonparametrics and Time Series, (Edited by S.Ghosh), Marcel Dekker, New York, 1999, 757-788. CMP 2000:05

10. Sen, P.K.: A Hajek-Renyi inequality for stochastic vectors with applications to simultaneous confidence regions, Ann. Math. Statist., 42 (1971), 1132-1134.

11. Shixin, Gan.: The Hajek-Renyi inequality for Banach space valued martingales and the $p$ smoothness of Banach spaces, Statist. Probab. Lett., 32 (1997), 245-248. MR 97k:60006

12. Whittle, P.: Refinements of Kolmogorov's inequality, Theory Probab. Appl., 14 (1969), 310311. MR 39:7657]

Indian Statistical Institute, 7, SJSS Marg, New Delhi 110 016, India

E-mail address: blsp@isid.ac.in 